\title{
ORIENTACIONES DE META Y MOTIVACIONES EN LA PRÁCTICA FÍSICO-DEPORTIVA EN EL MEDIO NATURAL: RUTA DE PEREGRINAJE
}

Granero-Gallegos, Antonio; Baena-Extremera, Antonio; Ruiz-Montero, Pedro Jesús; Baños, Raúl ORIENTACIONES DE META Y MOTIVACIONES EN LA PRÁCTICA FÍSICO-DEPORTIVA EN EL MEDIO NATURAL: RUTA DE PEREGRINAJE

MHSalud, vol. 15, núm. 1, 2018

Universidad Nacional, Costa Rica

Disponible en: http://www.redalyc.org/articulo.oa?id=237056016003

DOl: https://doi.org/10.15359/mhs.15-1.2

Esta obra está bajo una Licencia Creative Commons Atribución-NoComercial-SinDerivar 3.0 Internacional. 


\section{ORIENTACIONES DE META Y MOTIVACIONES EN LA PRÁCTICA FÍSICO-DEPORTIVA EN EL MEDIO NATURAL: RUTA DE PEREGRINAJE \\ ORIENTATION OF THE MOTIVATIONS TO PRACTICE PHYSICAL ACTIVITY IN NATURAL ENVIRONMENT \\ ORIENTAÇÕES DE OBJETIVOS E MOTIVAÇÕES NA PRÁTICA FÍSICO-ESPORTIVA NO MEIO NATURAL: ROTA DE PEREGRINAJE}

Antonio Granero-Gallegos

Universidad de Almería, España

agranero@ual.es

iD http://orcid.org/ 0000-0002-1385-8386

\section{Antonio Baena-Extremera}

Universidad de Granada, España

abaenaextrem@ugr.es

iD http://orcid.org/ 0000-0002-3597-4038

Pedro Jesús Ruiz-Montero

Universidad de Granada, España

pedrorumo@ugr.es

(iD http://orcid.org/0000-0001-9349-2478

\section{Raúl Baños}

Universidad Autónoma Baja California, México

raulfb89@gmail.com

iD http://orcid.org/0000-0003-4261-4919
DOI: https://doi.org/10.15359/mhs.15-1.2

Redalyc: http://www.redalyc.org/articulo.oa? $\mathrm{id}=237056016003$

Recepción: 17 Enero 2018

Aprobación: 31 Mayo 2018

\section{Resumen:}

En este trabajo se han analizado las relaciones entre las orientaciones de meta y las motivaciones de senderistas y cicloturistas mayores de 15 años para practicar actividad físico-deportiva por el medio natural, en el contexto del Camino de Santiago. La investigación se ha desarrollado mediante la aplicación de un cuestionario autoadministrado a una muestra de un total de 1071 sujetos. Tras el análisis factorial, los resultados han puesto de manifiesto la importancia de la situación y la correspondencia entre el carácter intrínseco de las motivaciones relacionadas con el ocio, recreación y afiliación, así como con la evasión y válvula de escape, y la orientación a la tarea. La disposición motivacional al ego resulta menos relevante en las actividades físico-deportivas y recreativas en el medio natural, aunque, en este entorno, son aspectos que resultan más importantes para las mujeres. Como conclusión, motivaciones de índole espiritual, cultural, deportivo y de relación con otras personas, son los destacados por los peregrinos del Camino de Santiago.

Palabras clave: Camino de Santiago, motivación, actividad físico-deportiva.

\section{ABSTRACT:}

This paper analyses the relationships between the goal orientations and the hikers and cycle\#tourers' motivations older than 15 have to practice physical-sport activity in natural environments in the context of the Way of Saint James. The investigation has been developed by administering a self-administered questionnaire to a sample of 1.071 individuals. After the factorial analysis, the results have shown the importance of the situation and the relationship between the intrinsic character of the motivations related to leisure, recreation, and affiliation, as well as to the escape and escape valve, and the task orientation. The ego orientation is less outstanding in the physical-sport and recreational activities in the natural environment, although these are aspects that are more important for women in this environment.

KEYWORDS: Way of Saint James, motivation, sport and physical activity.

\section{Resumo:}


Neste trabalho analisaram-se as relações entre as orientações de objetivos e as motivações de caminhantes e ciclistas com mais de 15 anos de idade para a prática de atividade física junto a natureza, no contexto do Caminho de Santiago. A pesquisa foi desenvolvida aplicando-se um questionário autoadministrado a uma amostra de 1.071 sujeitos. Depois da análise fatorial, os resultados mostraram a importância da situação e a relação entre a natureza intrínseca das motivações relacionadas ao lazer, recreação e adesão, bem como a evasão e a válvula de escape, e a orientação à tarefa. A orientação ao ego resulta menos relevante em as atividades físico-esportivas e recreativas junto a natureza, ainda que, neste meio, são aspectos que resultam mais importantes para as mulheres. Como conclusão, motivações de índole espiritual, cultural, esportivo e de relação com outras pessoas, são as destacadas pelos peregrinos do Caminho de Santiago.

PalaVras-Chave: Caminho de Santiago, motivação, atividade físico-esportiva.

\section{INTRODUCCIÓN}

La motivación es un término global usado para definir un factor psicológico central que interviene en la percepción de la fatiga (Noakes, 2012). Según Weman-Josefsson, Johnson y Lindwall (2018), se trata de un complejo proceso individual que incluye motivación intrínseca (cuando el compromiso es producido por el interés) y una regulación identificada por valores personales. En el análisis, la motivación se refiere a aquellos factores de la personalidad, variables sociales y cogniciones que entran en juego cuando la persona realiza una tarea. Aunque puede haber prioridad de unas razones sobre otras, la naturaleza multifacética de este constructo expone la existencia múltiple de ellas para viajar y hacer ejercicio en el medio natural (Newland, Newton, Stark, Podlog \& Hall, 2017). De forma semejante, afirman Weed y Bull (2004), en la práctica deportiva intervienen varios impulsos, por ejemplo: la recuperación psicológica, el escape a la rutina diaria y el sentimiento de libertad. Sin embargo, algunas de estas razones son mucho más específicas y contienen un marcado carácter deportivo-competitivo: la necesidad de competir, el deseo de ganar y el desarrollo de los niveles de destreza y habilidad.

Las diversas formas de afrontar una misma tarea que se acaban de exponer, se corresponden con dos concepciones diferentes en cuanto a las motivaciones, dentro de la teoría de las metas de logro: la orientación a la tarea y al ego. Esta perspectiva ha sido ampliamente investigada tanto en el ámbito de la educación física como en el deporte (Isorna, Rial, \& Alacid, 2011), analizando las variables más relevantes en el nivel cognitivo, emocional y afectivo. Cuando el éxito en el deporte se busca a través del esfuerzo, de la mejora y el desarrollo personal se conoce como orientación a la tarea y se asocia a patrones más adaptativos. Una concepción de habilidad consistente en juzgar la competencia personal en comparación con quienes se practica, sintiéndose exitoso cuando se demuestra superior habilidad que los demás se denomina orientación al ego (Isorna et al., 2011).

Sin embargo, la perspectiva de las metas de logro considera que la orientación de metas en el nivel personal (ego o tarea) pueden ser moduladas e influidas por el entorno que percibe el sujeto (Vallerand \& Rousseau, 2001). Así, la probabilidad de utilizar una u otra concepción de capacidad depende tanto de factores personales como de situación; no obstante, existen diferencias de carácter individual en la tendencia hacia uno u otro tipo de implicación (Harwood \& Swain, 2001).

De tal manera que la investigación relativa a las variables motivacionales asociadas a la orientación de las metas ha mostrado que la orientación a la tarea se ha ligado a una mayor motivación intrínseca (se refiere a los beneficios y satisfacciones inherentes a la propia actividad: el deseo por lo que se hace, la diversión en la práctica deportiva, la relación social, etc.), la orientación al ego se ha asociado con una búsqueda de recompensas extrínsecas a la tarea en sí (razones no directamente vinculadas con el desarrollo de la actividad: ganar y obtener recompensas, demostrar su capacidad a los demás) (López-Walle, Balaguer, Lluís Meliá, Castillo, \& Tristán, 2011). Cuando la actividad deportivo-recreativa se desarrolla en la naturaleza, en periodos vacacionales u ocupación de tiempo libre y como actividad de ocio, algunos autores (GraneroGallegos, 2010; Granero-Gallegos y Baena-Extremera, 2011) afirman que lo que se busca es escapar de las grandes urbes y cambiar de escenario, eliminar las tensiones y el estrés propios de la rutina de la vida diaria, 
relajarse, practicar actividad física que compense un estilo de vida inactivo, aventura, ocupar activamente el tiempo de ocio y el periodo vacacional, la realización personal y la relación con otras personas. En definitiva, los "aventureros diarios" en la naturaleza, como los denominan estos autores, buscan cada vez beneficios más numerosos y diversificados. Muchos de estos practicantes persiguen la experiencia emotiva y la aventura efímera; el hedonismo que se ha convertido en el eje principal y el epicentro de la cultura posmoderna. Otros, por el contrario, prefieren la competencia, el desafío y el riesgo. Teniendo en cuenta la citada teoría de las perspectivas de las metas, dentro de la teoría social-cognitiva, en el presente estudio partimos de la base, dadas las características de este itinerario cultural por el medio natural -entorno-, de que los motivos para afrontar un recorrido como el del Camino de Santiago están orientados hacia la tarea, asociados a una mayor motivación intrínseca, según la teoría desarrollada por Deci y Ryan (1985), pues se persigue el propio desarrollo y enriquecimiento personal, así como satisfacciones inherentes a la propia actividad.

De esta forma, una posible hipótesis sería que las motivaciones de carácter religioso, de ocio, recreación y afiliación y, evasión y válvula de escape se relacionan con una orientación a la tarea en aquellos que realizan el peregrinaje del Camino de Santiago. Por el contrario, las motivaciones hacia la demostración de capacidad se orientan hacia el ego. Asimismo, el objetivo de este estudio es doble: en primer lugar se estudiarán los motivos de senderistas y cicloturistas para recorrer el Camino de Santiago, identificando las posibles dimensiones; en segundo lugar, analizar las relaciones entre los distintos factores motivacionales de los viajeros jacobeos como practicantes de actividad físico-deportiva por el medio natural y las perspectivas de meta, según la variable sexo.

\section{MÉTODO}

\section{Muestra}

La investigación planteada está constituida por los peregrinos mayores de 15 años que inician en Roncesvalles su recorrido del Camino de Santiago en España, bien sea su lugar de salida o el primer lugar de España en el que queda constancia de su paso si provienen de Francia (senderistas y cicloturistas). En este trabajo se asume, para la validez en los resultados, un margen de error muestral del $\pm 3 \%$ y un nivel de confianza del $95.5 \%$. Así, se considera que la muestra representativa es de 1071 sujetos.

El diseño de la investigación se basa en proporciones y utiliza, para la elección de la muestra, el muestreo estratificado polietápico con afijación proporcional, dividiéndola por estratos o cuotas, en distintas etapas, según las características de la población (Tabla 1). Debido a la heterogeneidad de la muestra que compone este estudio (edad, sexo, medio de locomoción y nacionalidad), la estratificación permite que todas las personas que componen la población sean susceptibles de ser seleccionados para la muestra. En el caso del presente trabajo, indicar que la proporción de participación según sexo fue: $60.8 \%$ de varones (652) y el $39.2 \%$ de mujeres (419). Para la estratificación de la muestra se han tenido en cuenta los datos proporcionados desde el Archivo de la Real Colegiata de Roncesvalles. 
TABLA 1

Distribución de la muestra por edad, sexo, medio locomoción y nacionalidad

\begin{tabular}{|c|c|c|c|c|c|c|c|c|c|c|c|c|}
\hline \multirow[b]{3}{*}{ Edad (años) } & \multicolumn{6}{|c|}{ A pie } & \multicolumn{6}{|c|}{ En bicicleta } \\
\hline & \multicolumn{3}{|c|}{ Varones } & \multicolumn{3}{|c|}{ Mujeres } & \multicolumn{3}{|c|}{ Varones } & \multicolumn{3}{|c|}{ Mujeres } \\
\hline & Esp & Eur & $\mathrm{Rm}$ & Esp & Eur & $\mathrm{Rm}$ & Esp & Eur & $\mathrm{Rm}$ & Esp & Eur & $\mathrm{Rm}$ \\
\hline 16 a 20 & 33 & 33 & 7 & 23 & 22 & 6 & 8 & 7 & 2 & 5 & 5 & 1 \\
\hline 21 a 30 & 68 & 67 & 17 & 44 & 43 & 11 & 15 & 14 & 3 & 9 & 9 & 2 \\
\hline 31 a 40 & 47 & 47 & 11 & 31 & 30 & 7 & 11 & 10 & 2 & 7 & 7 & 1 \\
\hline 41 a 50 & 40 & 40 & 10 & 27 & 26 & 6 & 10 & 9 & 2 & 6 & 6 & 1 \\
\hline 51 a 60 & 33 & 32 & 7 & 21 & 20 & 5 & 7 & 7 & 2 & 4 & 4 & 0 \\
\hline$>\operatorname{de} 60$ & 19 & 18 & 4 & 12 & 12 & 2 & 4 & 4 & 0 & 2 & 2 & 0 \\
\hline Total & 240 & 237 & 56 & 158 & 153 & 37 & 55 & 51 & 12 & 33 & 33 & 5 \\
\hline
\end{tabular}

\section{Instrumento}

Se utilizó la dimensión referente a las motivaciones para recorrer el Camino de Santiago del Cuestionario para el Análisis de las Expectativas, Hábitos de Actividad Físico-Deportiva y Estilo de Vida (CAEHAFDEV), que desarrolló Granero-Gallegos (2005) para su investigación y cuya fiabilidad y validez demostró en su trabajo.

Se desarrolló el trabajo de campo en la Oficina del Peregrino de Roncesvalles, recogiendo la información mediante la aplicación de forma autoadministrada del cuestionario CAEHAFDEV diseñado por GraneroGallegos (2005) y en cuyo estudio se demuestra su fiabilidad y validez

\section{Procedimiento}

Se contactó con la Oficina de Peregrinos de la Real Colegiata de Roncesvalles, para solicitar los datos referentes a las personas que recorrían el Camino de Santiago. Así mismo, se pidió su colaboración para la recolección de los datos mediante el cuestionario auto-administrado, en la propia oficina, a todos aquellos peregrinos y peregrinas que pasaban por la misma y en la que queda primera constancia del paso o inicio del Camino de Santiago en su recorrido por España. Se informa a las personas responsables en la Real Colegiata de Roncesvalles de las intenciones y de los objetivos de la investigación y deciden colaborar con la investigación. Durante la recogida de la información, cada uno de los participantes son informados del objetivo de estudio, voluntariedad y confidencialidad de las respuestas y el manejo de datos, se les indica que no había respuestas correctas o incorrectas. Durante el proceso de aplicación del cuestionario se resuelve cualquier duda surgida respecto al significado de los ítems. La recogida de los instrumentos se realiza individualmente, de esta manera se pueden detectar errores y verificar que ninguno queda en blanco. Así mismo, solo se recogieron datos de aquellas personas menores de edad que iban acompañadas por su padre/madre/tutor, que tuvieron que firmar una autorización para poder participar en la investigación. Debido que una de las cuotas de la investigación es la nacionalidad, se tradujo el cuestionario a cinco idiomas distintos: español, inglés, francés, italiano y alemán.

\section{Análisis de datos}

Se ha realizado un análisis factorial de la pregunta que recoge los motivos de los viajeros jacobeos para recorrer este itinerario por el medio natural, el Camino de Santiago. Indicar, además, que se les ofreció la posibilidad de que marcaran todas las razones que estimaran oportunas, no solo una de ellas. Para el estudio de las diferencias 
por sexo se ha utilizado la prueba de $\mathrm{T}$ de Student, y se consideran estadísticamente significativos los valores $\mathrm{p}<0,05$. Todo ello realizado con el SPSS 22.0.

\section{RESULTADOS}

\section{Estructura factorial de las motivaciones}

Para determinar la estructura factorial de los motivos para recorrer el Camino de Santiago se ha realizado un análisis de los componentes principales. El criterio aplicado para decidir el número de factores a rotar se ha basado en aquellos factores con un autovalor $>$ a 1 . La validez de constructo se examinó a través de análisis factoriales de componentes principales con rotaciones Varimax, requiriéndose una correlación mínima del .45 para considerar una variable importante dentro de un factor (Tabla 2).

TABLA 2

Análisis factorial. Matriz de componentes rotados

\begin{tabular}{llll}
\hline & 1 & 2 & 3 \\
\hline Vacaciones y turismo & .766 & & \\
Por diversión & .755 & & \\
Hacer actividad física & .704 & & \\
Aventura & .615 & & \\
Relacionarme con gente y hacer amigos & .474 & & \\
Búsqueda de tranquilidad & & .722 & \\
Búsqueda interior, espiritualidad & & .670 & \\
Alejarme de la vida cotidiana & & .606 & .715 \\
Experiencia personal y vivencial & & .501 & .647 \\
Reto personal & & & .757 \\
Competición & & & .727 \\
Religiosos & & & \\
Conseguir la "Compostela" & & \\
\hline
\end{tabular}

En la Tabla 2 se muestran los resultados del análisis factorial de las variables que miden las motivaciones que mueven a los senderistas y cicloturistas por el Camino de Santiago en la actualidad. La agrupación de variables ha ofrecido cuatro factores. El primero agrupa las variables relativas al ocio, recreación y afiliación, incluyendo las respuestas: "vacaciones y turismo", "por diversión”, "hacer actividad física”, "aventura” y "relacionarme con gente y hacer amigos". El segundo factor recoge los motivos relacionados con la evasión y válvula de escape: "búsqueda de tranquilidad", "búsqueda interior y espiritualidad", "alejarme de la vida cotidiana" y "experiencia personal y vivencial". El tercer factor recoge las variables que hacer referencia a la demostración de capacidad, tales como "reto personal" y "competición". Finalmente, el cuarto factor agrupa los motivos más relacionados con el carácter tradicional de recorrido de esta ruta jacobea: "religiosos" y "conseguir la Compostela”. En su conjunto las cuatro dimensiones de motivación explican el 52,8 \% de la varianza.

El coeficiente $\mathrm{KMO}$ es de .788, lo que indica el alto grado de adecuación muestral, pues el grado de variabilidad total de la muestra se explica al $78.8 \%$. Asimismo, el nivel crítico de la prueba de esfericidad de Bartlett indica el alto grado de correlaciones positivas entre variables. La consistencia interna de las dimensiones de motivación ha sido medida a través de la Alfa de Cronbach, siendo los resultados aceptables en los tres primeros factores (alfa $=.83$ en el factor ocio, recreación y afiliación; .72 en el denominado evasión y válvula de escape; .68 en el de demostración de capacidad), mientras que en el cuarto, el que hace referencia a las motivaciones de carácter tradicional, el resultado de fiabilidad es bajo (alfa $=.30$ ). Por esta razón, no se van a analizar las diferencias que se producen en relación con esta última dimensión. 


\section{Diferencias en función de la variable sexo}

Como se observa en la Tabla 3, existen significativas diferencias en función de la variable sexo. Llama la atención que en relación con las tres dimensiones estudiadas, la mujer presenta valores superiores a la media, mientras que los varones lo hacen por debajo (Tabla 3). Asimismo, la prueba $T$ para la igualdad de medias muestra que se producen diferencias significativas entre unas y otros, respecto a las motivaciones de evasión y válvula de escape $(\mathrm{t}=15.31 ; \mathrm{p}<.001)$.

TABLA 3

Prueba $\mathrm{T}$ de muestras independientes según la variable sexo

\begin{tabular}{lcccccccc}
\hline \multicolumn{1}{c}{ Componentes } & Varón (n=639) & \multicolumn{2}{c}{$\begin{array}{c}\text { Mujer } \\
(\mathbf{n = 4 0 0 )}\end{array}$} & \multicolumn{3}{c}{$\begin{array}{c}\text { Prueba t de Student } \\
\text { Significac. }\end{array}$} & $\begin{array}{c}\text { Diferencia de } \\
\text { medias }\end{array}$ \\
\hline Ocio. recreación y afiliación & -.043 & 1.005 & .069 & .990 & 3.109 & .078 & .112 \\
Evasión y válvula de escape & -.095 & 1.007 & .152 & .971 & 15.313 & .000 & .248 \\
Demostración de capacidad & -.004 & 1.097 & .006 & .824 & .022 & .882 & .093 \\
\hline
\end{tabular}

Nota: $\mathrm{M}=$ Media sobre valores tipificados; $\mathrm{DT}=$ Desviación típica; $p$ es significativo al valor $<.05$

\section{DISCUSIÓN}

Los objetivos de esta investigación fueron dos, por un lado se estudió los motivos de los senderistas y cicloturistas para recorrer el Camino de Santiago, identificando las posibles dimensiones; en segundo lugar, se analizó las relaciones entre los distintos factores motivacionales de los viajeros jacobeos como practicantes de actividad físico-deportiva por el medio natural y las perspectivas de meta, según la variable sexo. La importancia de esta investigación radica en cuáles son los motivos que llevan a las personas a emprender un perseverante peregrinaje de días, semanas e incluso meses.

En relación al primer objetivo, tanto la dimensión de ocio, recreación y afiliación como la de evasión y válvula de escape, se asocian a un mayor interés intrínseco, pues a través de estas motivaciones se pretende el propio desarrollo y enriquecimiento personal, así como satisfacciones propias de la actividad. La diversión, que en este caso forma parte de la primera dimensión citada, aparece como concepto clave, tanto en la orientación a la tarea como en la percepción de un clima motivacional hacia la tarea, en los estudios de Cervelló, Escartí y Balagué (1999) y Duda y Nicholls (1992). Por el contrario, la demostración de capacidad se relaciona con la disposición al ego, pues se persigue una recompensa extrínseca.

Aunque en esta investigación, no se ha analizado el factor carácter tradicional que se relaciona con la religiosidad, cabe destacarlo por ser uno de los principales motivos por el que los peregrinos realizan el Camino de Santiago, convirtiéndose en meta de peregrinación cristiana al mismo nivel que Roma y Jerusalén según García y Palma (2017). En esta línea, Montes (2015) y Sloterdijk (2012), destacan la convivencia de motivaciones entre lo religioso y el turismo especial a la hora de realizar la peregrinación, además del patrimonio cultural y los elementos arquitectónicos (Lois \& López, 2015), destacan como motivos de peregrinaje el turismo,

En relación al segundo objetivo, las mujeres se sienten significativamente más identificadas con los motivos de evasión y válvula de escape, pues parece que afrontan este tipo de actividades tratando de alejarse del mundo laboral y del peso de las responsabilidades familiares. Algunos autores confirman las teorías acerca de la búsqueda de emociones "lúdicas" y motivos para la autorrealización, pues los datos evidencian la importancia de las variables relacionadas con la evasión y válvula de escape. Por ello, la escasez de tiempo y la presión del día a día supone para ellas, que las actividades de estas características les faciliten una 
"desconexión" con el mundo cotidiano, buscando escape y evasión, aunque sea momentánea (GraneroGallegos, Ruiz-Juan, \& García-Montes, 2007). Baena-Extremera y Rebollo (2009), encontraron la diversión, aventura personal y las relaciones sociales, como principales motivos por los que las mujeres practican deportes en la naturaleza, siendo la competición otra variable que las impulsa al medio natural. Por el contrario, los principales motivos por los que no participan en deportes de la naturaleza son por la falta de educación deportiva que han recibido ellas, la cultura deportiva y la dificultad física (Baena-Extremera \& Rebollo, 2008).

Respecto a las orientaciones disposicionales de meta, investigaciones realizadas en el ámbito deportivo (Castillo, Balaguer, \& Duda, 2000) exponen el mayor interés de los chicos en ganar y demostrar su capacidad en los contextos competitivos que las chicas. Así, dadas las características físico-recreativas de esta actividad y el reseñado sentido de autorrealización y desrutinización, es menor la cantidad de peregrinos que se sienten atraídos por la competición (Granero-Gallegos et al., 2007). Además, la mujer siente más la necesidad de autorrealización, de tranquilidad y alejamiento de la rutina cotidiana. Y es que en su vida diaria soportan gran presión (Granero-Gallegos et al., 2007), acostumbradas a responder continuamente a las necesidades y demandas de otros, tanto en la soledad del hogar como en el trabajo, donde habitualmente, además, ocupan puestos subordinados.

Es manifiesta la importancia de la situación y la relación entre el carácter intrínseco de las motivaciones expresadas y la orientación a la tarea de las mismas, dada la búsqueda de la satisfacción personal, experimentando sensaciones diferentes a las cotidianas mediante la realización activa de estas prácticas de actividad físico-deportiva que sirven de vínculo con la naturaleza y de evasión y válvula de escape del día a día. Mientras que, la orientación al ego o demostración de capacidad resulta menos relevante en este tipo de actividades en el medio natural.

\section{CONCLUSIÓN}

Cabe destacar que las motivaciones de los peregrinos son diferentes y argumentan diversidad de razones, combinándose las de índole espiritual, las culturales, las deportivas y las de relación con otras personas. El arte y la cultura de la ruta, hacer actividad física, relacionarse con gente, hacer amigos y vivir una aventura, son motivaciones para la mayoría de los que recorren el Camino de Santiago. Además, las vacaciones y el turismo constituyen una razón destacada, prácticamente para la mitad de los peregrinos entrevistados en el itinerario jacobeo. También, destacan el alejarse de la vida cotidiana y vivir una importante experiencia personal, de búsqueda interior y espiritualidad como motivos del porqué hacer la peregrinación.

En cuanto a las orientaciones de meta, las dimensiones de ocio, recreación y afiliación y la de evasión y válvula de escape, se relacionan con una disposición hacia la tarea, ya que se asocian a patrones más adaptativos, buscando la superación personal y el esfuerzo de la mejorar. Por el contrario, la dimensión demostración de capacidad, se vincula metas orientadas al ego, cuyo objetivo es demostrar la competencia personal en comparación con los demás, consiguiendo el éxito cuando se demuestra superior capacidad que sus iguales. Para finalizar, no se hallaron resultados significativos en la dimensión de carácter tradicional.

\section{REFERENCIAS}

Baena-Extremera, A., y Rebollo, S. (2008). Análisis del perfil sociodemográfico de la mujer como participante en raids de aventura (Social-demographic analysis of raids adventure sportswomen). Retos, 14, 30-34.

Baena-Extremera, A., y Rebollo, S. (2009). Análisis del perfil deportivo de la mujer como practicante de raids de aventura. Revista Española de Educación Física y Deportes, 386, 65-78.

Castillo, I., Balaguer, I. y Duda, J. L. (2000). Las orientaciones de meta y los motivos de práctica deportiva en los jóvenes deportistas valencianos escolarizados. Revista de Psicología del Deporte, 1-2, (9), 37-50. 
Cervelló, E., Escartí, A., y Balagué, G. (1999). Relaciones entre la orientación de meta disposicional y la satisfacción con los resultados deportivos, las creencias sobre las causas de éxito en deporte y la diversión con la práctica deportiva. Revista Psicología del Deporte, 8, (1), 7-19.

Deci, E. L., \& Ryan, R. M. (1985). Intrinsic motivation and self-determination in human behaviour. New York: Plenum Ples. https://doi.org/10.1007/978-1-4899-2271-7

Duda, J. L., \& Nicholls, J. G. (1992). Dimensions of achievement motivation in schoolwork and sport. Journal of Educational Psychology, 84, 1-10. https://doi.org/10.1037/0022-0663.84.3.290

García, A. M., \& Palma, S. S. (2017). Aragón: Grail territory. International Journal of Scientific Management and Tourism, 3(4), 467-494.

Granero-Gallegos, A. (2005). Expectativas y vivencias en la actividad físico-deportiva del peregrino. Un antes y un después en el Camino de Santiago [CD ROM]. Almería: Universidad de Almería. Servicio de Publicaciones.

Granero-Gallegos, A. (2010). El Camino de Santiago. Guía de vivencias del Peregrino del siglo XXI. Sevilla: Wanceulen.

Granero-Gallegos, A., \& Baena-Extremera, A. (2011). The sport systems a reflection the social enviroment. Journal of Sport and Health Research, 3(3), 211-228.

Granero-Gallegos, A., Ruiz-Juan, F., García-Montes, M. E. (2007). Estudio sobre las motivaciones para recorrer el Camino de Santiago. Apunts, Educación Física y Deportes, 89, 88-96.

Harwood, C., \& Swain A. (2001). The development and activation of achievement goals in tennis: I. Understanding the underlying factors. Sport Psychology, 1, 319-341. https://doi.org/10.1123/tsp.15.3.319

Isorna, M., Rial, A., \& Alacid, F. (2011). Análisis de la teoría de metas de logro y de la autodeterminación en piragüistas de élite. Revista Galega de Cooperación Científica Iberoamericana, 21, 15-23.

Lois, R. \& López, L. (2015). El origen del turismo viajero italiano a lo largo del Camino de Santiago. Investigaciones Turísticas, 9, 132-159.

López-Walle, J., Balaguer, I., Lluís Meliá, J., Castillo, I., \& Tristán, J. (2011). Adaptación a la población mexicana del Cuestionario de Orientación al Ego y a la Tarea en el Deporte (TEOSQ). Revista de Psicología del Deporte, 20(2), 523-536.

Montes, C. (2015). Al andar se hace turismo: nuevas ruralidades en torno al Camino de Santiago. Aposta. Revista de Ciencias Sociales, 65, 131-150.

Newland, A., Newton, M., Stark, A., Podlog, L. \& Hall, M. (2017). College student's perception of a caring climate in group physical activity classes. Biomedical Human Kinetics, 9, 99-106. https://doi.org/10.1515/ bhk-2017-0015

Noakes, T. D. (2012). Fatigue is a brain-derived emotion that regulates the exercise behaviour to ensure the protection of whole body homeostasis. Frontiers in Physiology, 3, 82. https://doi.org/10.3389/fphys.2012.00082

Sloterdijk, P. (2012). Has de cambiar tu vida. Sobre antropotécnica. Valencia, Pretextos.

Vallerand, R. J., \& Rousseau, F. L. (2001). Intrinsic and extrinsic motivation in sport and exercise: A review using the hierarchical model of intrinsic and extrinsic motivation. In R. N. Singer, H. A. Hausenblas, \& C. M. Janelle (Eds.), Handbook of sport psychology (2nd ed., pp. 389-416). New York: Wiley.

Weed, M., \& Bull, C. (2004). Sports Tourism. Oxford: Butterworth-Heinemann.

Weman-Josefsson, K., Johnson, U., \& Lindwall, M. (2018). Zooming in on the Effects: a Controlled Trial on Motivation and Exercise Behaviour in a Digital Context. Current Psychology, 37(1), 250-262. https:// doi.org/10.1007/s12144-016-9508-1

\section{BY-NC-ND}

